\title{
Correction to: GeNeDis 2018: Computational Biology and Bioinformatics
}

\author{
Panayiotis Vlamos
}

\section{Correction to: \\ P. Vlamos (ed.), GeNeDis 2018, Advances \\ in Experimental Medicine and Biology 1194, https://doi.org/10.1007/978-3-030-32622-7}

In the original version of this book, Chapter 20 was inadvertently published without carrying the corrections provided by the author. This has now been rectified in this revised version of the book.

All of the citations are corrected due to a string-related error.

The sentence: "After the introduction in Sects. 1 and 2 presents former related work in the area, and Sect. 3 includes the basic definitions and notations that are needed" has been updated to: "After the introduction in Section 1, Section 2 presents former related work in the area and Section 3 includes the basic definitions and notation that are needed".

Chapter 46 was inadvertently published with few errors in Table 2 data. This has now been rectified in this revised version of the book.

In Table 2, the random summary effect "- 0.55 " have been updated to "- 0.055 " and Choi2010 have been updated to Choi2014.

\footnotetext{
The updated online versions of these chapters can be found at https://doi.org/10.1007/978-3-030-32622-7_20 https://doi.org/10.1007/978-3-030-32622-7_46
} 